\title{
Circuit
}

Musiques contemporaines

\section{Discographie du NEM}

\section{Cléo Palacio-Quintin}

Volume 23, numéro 3, 2013

Géométries durables : pour les 25 ans du Nouvel Ensemble Moderne

URI : https://id.erudit.org/iderudit/1021520ar

DOI : https://doi.org/10.7202/1021520ar

Aller au sommaire du numéro

Éditeur(s)

Les Presses de l’Université de Montréal

ISSN

1183-1693 (imprimé)

1488-9692 (numérique)

Découvrir la revue

Citer ce document

Palacio-Quintin, C. (2013). Discographie du NEM. Circuit, 23(3), 75-89.

https://doi.org/10.7202/1021520ar

Ce document est protégé par la loi sur le droit d'auteur. L'utilisation des services d'Érudit (y compris la reproduction) est assujettie à sa politique d'utilisation que vous pouvez consulter en ligne.

https://apropos.erudit.org/fr/usagers/politique-dutilisation/
Cet article est diffusé et préservé par Érudit.

Érudit est un consortium interuniversitaire sans but lucratif composé de l'Université de Montréal, l'Université Laval et l'Université du Québec à Montréal. Il a pour mission la promotion et la valorisation de la recherche. https://www.erudit.org/fr/ 


\section{Discographie du NEM}

Cléo Palacio-Quintin

Depuis sa fondation en 1989, le Nouvel Ensemble Moderne (NEM) a réalisé des enregistrements pour 29 productions discographiques, en collaborant avec de multiples étiquettes de disques: ABC Classics, ATMA Classique, Amberola, Ambiances magnétiques, Auvidis-Naïve, Centredisques, Chants Libres, Composers Recordings Inc., Doberman-Yppan, Mittledeutscher Rundfunk, Montaigne, New World Record Inc., Stradivarius, UMMUS; et en produisant quelques disques sous sa propre étiquette ProNEM. Plusieurs de ces enregistrements ont donné lieu à des collaborations avec différents ensembles et organisations, comme l'Atelier de gamelan de l'Université de Montréal, Australian Broadcasting Corporation, Bang on a Can, Chants Libres, le Centre de musique canadienne, le Centre interdisciplinaire de recherche en musique, médias et technologie (CIRMMT), Espace musique, Festival Musica 93, GRAME - Centre national de création musicale (Lyon), Ircam/Centre Georges Pompidou (Paris), Festival de Dresde, Percussions de Strasbourg, Radio-France, Société Radio-Canada, Lorraine et Jean-Eudes Vaillancourt (pianistes) et Vidéographe, ainsi qu'avec les solistes Simon Aldrich (clarinette), Tim Brady (guitare électrique), Michel Ducharme (baryton), Yegor Dyachkov (violoncelle), David Lang (narrateur), Joseph Petric (accordéon), Jay Rozen (tuba), et Pauline Vaillancourt (soprano).

Ces productions comprennent $27 \mathrm{CD}$ et 2 DVD qui regroupent un total de 99 œuvres. La plus «ancienne» composition, Kammerkonzert de György Ligeti, date de 1969-1970, et la plus récente, Mutation de Denis Gougeon, de 2011. Ces nombreux enregistrements nous permettent d'écouter la musique de 76 compositeurs originaires de 25 pays, dont 26 compositeurs de nationalité canadienne. On y retrouve plusieurs générations de compositeurs contemporains, le doyen étant Elliott Carter (États-Unis, 1908-2012) et le plus jeune, Taylor Brook (Canada, 1985). D'ailleurs, afin de souligner cette incroyable variété de répertoire, lors de son $20^{\mathrm{e}}$ anniversaire en 2009 , le NEM a produit un CD promotionnel qui reprend des extraits de 42 œuvres d'autant de compositeurs. Les œuvres ont été choisies parmi les multiples disques alors existants. 
Tous ces enregistrements peuvent être partagés en quatre différents types de productions:

- 6 CD et un DVD sont consacrés au répertoire de compositeurs québécois (d'origine ou d'adoption);

- ${ }_{4} \mathrm{CD}$ sont consacrés à du répertoire international;

- 8 CD et un DVD regroupent les créations de compositeurs de multiples origines ayant participé au Forum international des jeunes compositeurs du NEM au fil des ans;

- 9 CD sont produits par différentes organisations, et le NEM y apparaît comme collaborateur pour une ou deux pistes.

\section{Répertoire de compositeurs québécois}

- 1991: Musiques de Montréal

- 1996: Gougeon - Lesage - Perron - Provost

- 2004: Panneton - Demers - Lesage - Evangelista

- 2004: Tim Brady: Playing Guitar: Symphony \#1

- 2006: Oesterle - Provost - Tremblay: À quelle heure commence le temps?

- 2010: Opéra féerie - L'eau qui danse, la pomme qui chante et l'oiseau qui dit la vérité

- 2012: Bouliane-Gougeon-Rea

Ces six CD et le DVD consacrés au répertoire québécois comprennent 21 œuvres de 15 compositeurs. La majorité de ces œuvres sont d'ailleurs des commandes du NEM (12 parmi les 21). Il faut souligner que les trois plus récents de ces $\mathrm{CD}$, produits sur l'étiquette ATMA Classique, ont tous reçu le prix Opus - Disque de l'année, musique moderne, contemporaine lors de leur parution. L'Opéra féerie de Gilles Tremblay, enregistré en spectacle par Espace Musique, la radio musicale de Radio-Canada, et ensuite immortalisé sur DVD par Chants Libres, avait obtenu le prix Opus - Événement musical de l'année lors de sa création. Deux des commandes du NEM endisquées ont aussi obtenu le prix Opus - Création de l'année, soit: À quelle heure commence le temps? (1999) de Gilles Tremblay et Mutation (2011) de Denis Gougeon. Cette dernière œuvre est d'ailleurs la plus récente pièce endisquée par le NEM lors d'un enregistrement à la Salle Claude-Champagne en janvier 2012. 


\section{Musiques de Montréal}

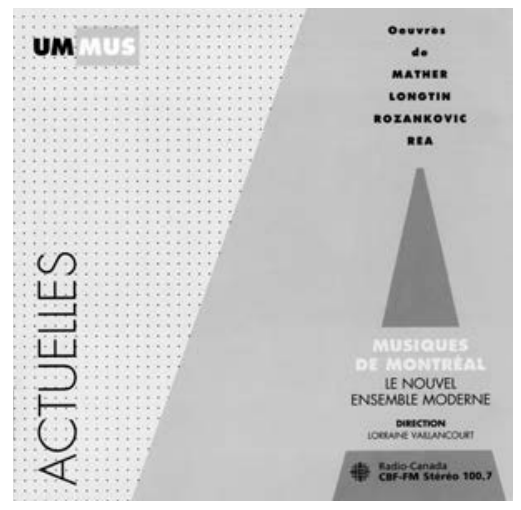

UMMUS / UMM 105 / 1991

Bruce Mather (1939)

- Travaux de nuit (1990)

Michel Longtin (1946)

- Colère: Berlin 61 (1989)

Anthony Rozankovic (1962)

- Pas de deux (1990)

John ReA (1944)

- Kubla Khan (1988)

Michel Ducharme (baryton)

Société Radio-Canada

\section{Gougeon - Lesage - Perron - Provost}

UMMUS / UMM 109/1996

Alain Perron (1959)

- Exil (1995)*

JEAN LeSAGE (1958)

- Les sensations confuses (1993)*

Denis Gougeon (1951)

- Un train pour l'enfer (1993)*

Serge Provost (1952)

- L'adorable verrotière (1992)

Percussions de Strasbourg Pauline Vaillancourt (soprano) Société Radio-Canada

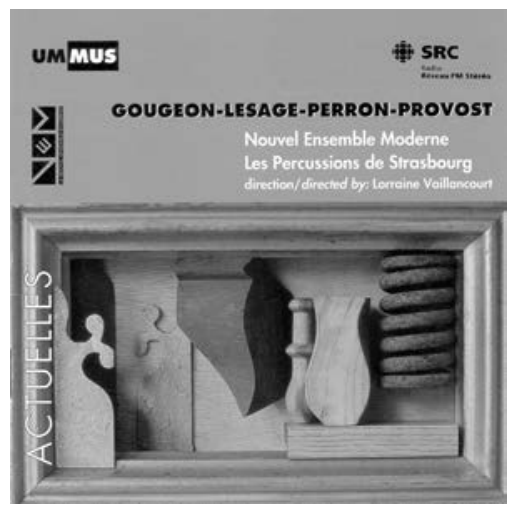

* Commandes du NEM

Panneton - Demers - Lesage - Evangelista

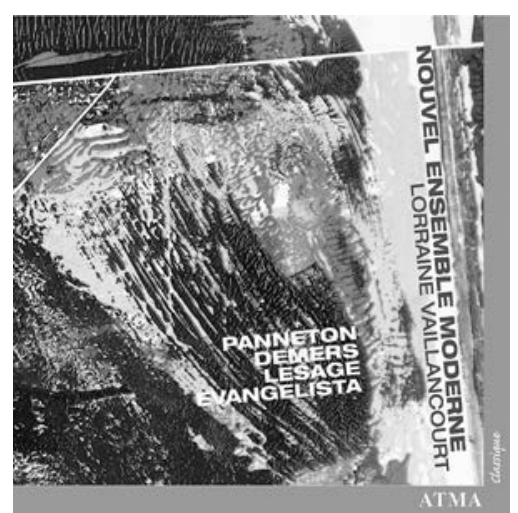

ATMA Classique / ACD2 2242 / 2004

IsABelle PANNeton (1955)

- Travaux et jeux de gravité (2000)*

INOUK DEMERS (1970)

- Lo que vendrá (2000)*

JeAn Lesage (1958)

PR I X
O PUS

- Vanitas (2002)

An 8-03/04

José EvangELISTA (1943)

- Alap \& Gat (1998)* 
Tim Brady: Playing Guitar: Symphony \#1

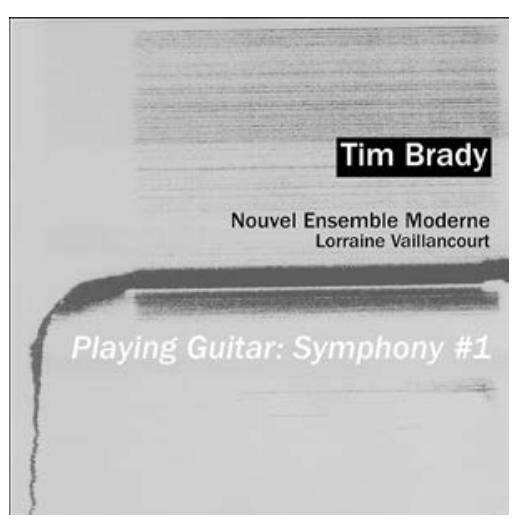

Ambiances magnétiques / AM 125/ 2004

Tim Brady (Canada, 1956)

- Playing Guitar: Symphony \#1 (1997-2000)*

Tim Brady (guitare électrique)

Oesterle - Provost - Tremblay À quelle heure commence le temps?

ATMA Classique / ACD2 2376 / 2006

Michael Oesterle (1968)

- Annus Mirabilis (2005)*

Serge Provost (1952)

\section{PRPU}

- Les ruines du paradis (2004)*

Gilles Tremblay (1932)

- À quelle heure commence le temps? (1999)*

Michel Ducharme (baryton)

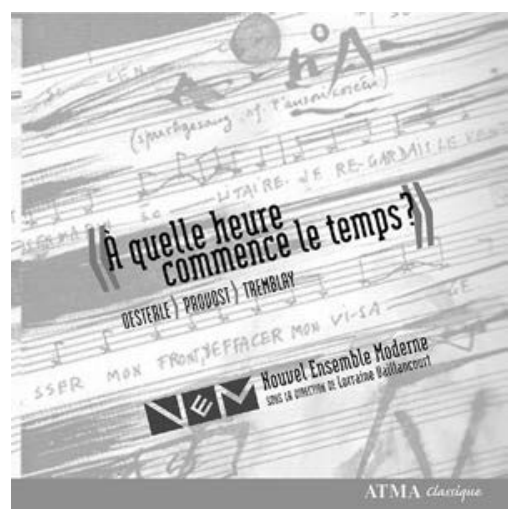

Opéra féerie - L'eau qui danse, la pomme qui chante et l'oiseau qui dit la vérité

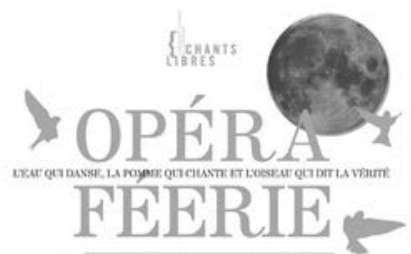

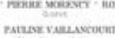
An $11-06 / 07$

Chants Libres / CL 2010

Gilles Tremblay (1932)

- Opéra féerie - L'eau qui danse, la pomme qui chante et l'oiseau qui dit la vérité (2009)

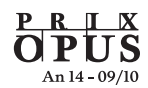

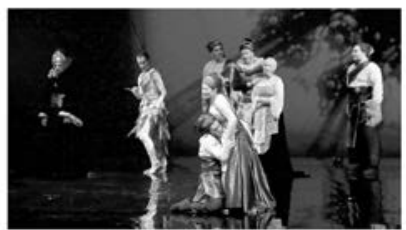




\section{Bouliane - Gougeon - Rea}

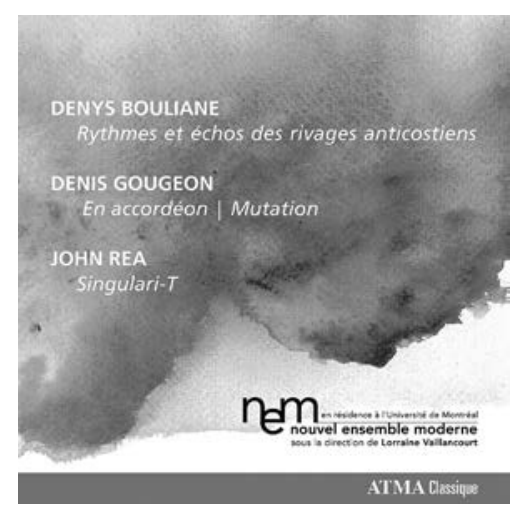

ATMA Classique / ACD2 2376 / 2012

Denys Bouliane (1955)

- Rythmes et échos des rivages anticos-

tiens (2009)

Denis Gougeon (1951)

- En accordéon (2004)

- Mutation (2011)

\section{PRPU}

JoHn REA (1944)

- Singulari-T (Tombeau de Ligeti) (2007)

Joseph Petric (accordéon)

\section{Répertoire international}

- 1990: Benjamin - Gentile - Saariaho - Ligeti

- 1996: Jonathan Harvey 2 : Bhakti

- 2000: Bhakti - Jonathan Harvey (réédition de l'enregistrement de 1996)

- 2001 : Elliott Carter

- 2005: Luis de Pablo

En plus de son intérêt pour le répertoire d'ici, le NEM s'est toujours positionné en défenseur de la diffusion du répertoire international. Leur tout premier CD, paru en 1990 sur l'étiquette UMMUS de la Faculté de musique de l'Université de Montréal - où l'ensemble est en résidence depuis sa fondation -, en témoigne. Il contient des œuvres de quatre compositeurs reconnus sur la scène internationale, soit: George Benjamin, Ada Gentile, Kaija Saariaho et György Ligeti. La directrice artistique souligne ainsi que «le NEM est un ensemble de répertoire, qui accueille les grands et les petits chefs-d'œuvre du XXe siècle ${ }^{1}$ ».

Les autres parutions de répertoire international sont des disques monographiques. L'œuvre Bhakti (1982) de Jonathan Harvey, d'une durée de près de 54 minutes, fait l'objet d'un CD complet dont l'enregistrement a été réalisé à l'Institut de recherche et coordination acoustique/musique (Ircam). Sacrée Diapason d'or, l'édition de 1996 sur l'étiquette Montaigne a rapidement été épuisée et le disque a fait l'objet d'une réédition avec la collaboration de Auvidis-Naïve en l'an 2000 (cette édition est également épuisée). L'année suivante, l'ensemble se fragmente en solo, duo, trio et triple duo pour immortaliser la musique de chambre de Elliott Carter, ainsi que son Concerto

1. Extrait du livret du disque Benjamin Gentile - Saariaho-Ligeti, UMMUS, UMM 102, 1990 (sans pagination). 
2. Extrait du livret du disque Luis de Pablo, ATMA Classique, ACD2 2353, 2005 (sans pagination) pour clarinette (1996) interprété par le clarinettiste du NEM Simon Aldrich. C'est le début d'une collaboration avec l'étiquette ATMA Classique qui présentera presque toutes les productions subséquentes du NEM. L'ensemble travaille ensuite avec Luis de Pablo pour endisquer trois œuvres de son répertoire. L'«inventif, fantaisiste, poétique, leader de l'école espagnole actuelle, $[\ldots]$ aujourd'hui l'une des personnalités les plus marquantes de la musique vivante ${ }^{2}$ » considère cette collaboration comme une de ses plus belles expériences.

\section{Benjamin - Gentile - Saariaho - Ligeti}

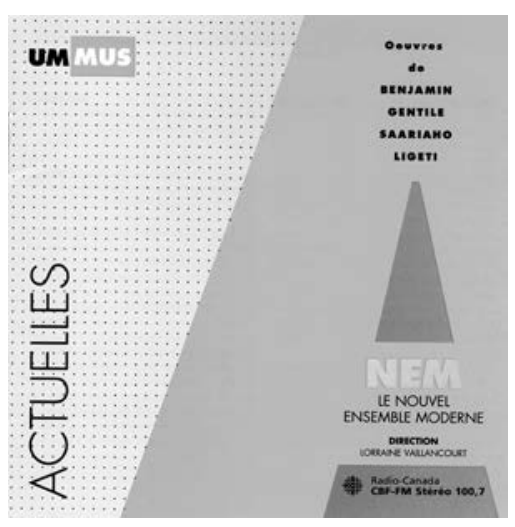

UMMUS / UMM 102 / 1990

George Benjamin (Angleterre, 1960)

- At first Light (1985)

Ada Gentile (Italie, 1947)

- In un silenzio ordinato (1985)

Kaija SaAriaho (Finlande, 1952)

- Lichtbogen (1985-1986)

GYÖRGY LIGETI (Roumanie, 1923-Autriche, 2006)

- Kammerkonzert (1969-1970)

\section{Jonathan Harvey 2: Bhakti}

Montaigne / MO 782086 / 1996

\section{Bhakti - Jonathan Harvey}

Montaigne/Auvidis-Naïve /

MO 782128 / 2000

JonATHAN HaRvey (Angleterre, 1939-2012)

- Bhakti (1982)

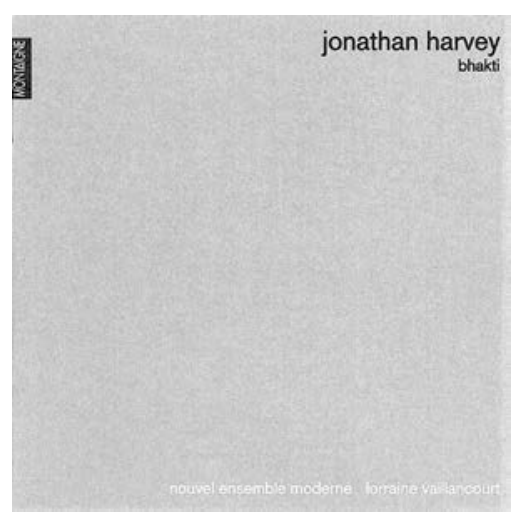




\section{Elliott Carter}

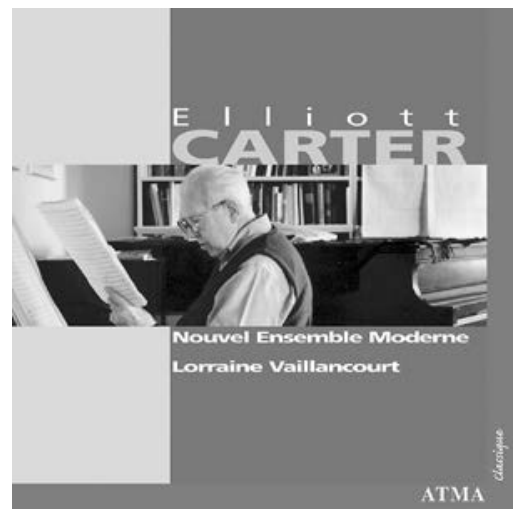

ATMA Classique / ACD2 2280 / 2001

Elliott CARTER (États-Unis, 1908-2012)

- Enchanted Preludes (1988)

- Con Leggerezza Pensosa (1990)

- Triple Duo (1982-1983)

- Gra (1993)

- Riconoscenza per Goffredo Petrassi (1984)

- $90+(1994)$

- Esprit rude / Esprit doux (1985)

- Esprit rude / Esprit doux II (1995)

- Concerto pour clarinette (1996)

\section{Luis de Pablo}

ATMA Classique / ACD2 2353/2005

Luis de PABLO (Espagne, 1930)

- Paraiso y tres danzas macabras

(1991-1992)

- Segunda lectura (1993)

- Razón dormida (2003)

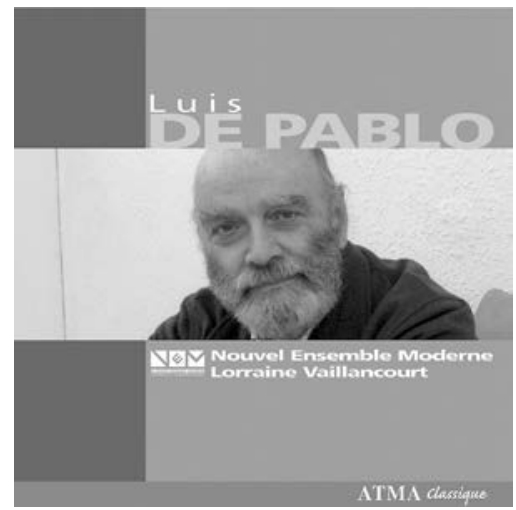

\section{Forum international des jeunes compositeurs}

- 1993: Forum 91

- 1995: Forum 93

- 1998: Forum 96

- 2000: Forum 98

- 2003: Forum 2000-2002

- 2005: Forum 2004

- 2008: Forum 2006 à Amsterdam

- 2009: Forum 2008 - Musique mixte

- 2012 : Forum 2010 - Musique et vidéo d'art - DVD

Depuis la première édition de 1991, il y a eu 11 Forums du NEM qui ont permis 
3. "Vidéographe est un centre autogéré d'artistes voué à la création, la diffusion et la distribution d'œuvres d'arts médiatiques indépendantes." Voir: $<$ www.videographe.qc.ca> (consulté le 14 octobre 2013). semaines intensives de travail en étroite collaboration avec l'ensemble. Huit CD (dont trois doubles CD) et un DVD ont été consacrés aux enregistrements de nombreuses œuvres créées pour l'occasion.

Dans le CD du tout premier Forum 91, on retrouve aussi une œuvre du compositeur canadien Brian Cherney, en plus de celles des trois jeunes compositeurs finalistes. Le CD du Forum 2006, qui a eu lieu à Amsterdam, comprend aussi, en plus des quatre œuvres des jeunes compositeurs ayant participé à cette édition, deux œuvres commandées par le NEM aux compositeurs Éric Morin (Canada) et Robin de Raaff (Pays-Bas).

Les autres disques et le DVD sont exclusivement dédiés aux œuvres créées pour les Forums. Au total, 46 jeunes compositeurs originaires de 23 pays ont eu le privilège de voir leur œuvre endisquée par le NEM. (Vous lirez plus bas leur nom, origine et année de naissance, ainsi que le titre de leur composition. L'ordre des pistes sur chaque CD est respecté.)

D'autre part, le double CD Forum 2000-2002 s'est distingué en recevant le prix Coups de cœur musique contemporaine de l'Académie Charles Cros.

L'édition du Forum 2010, consacrée à la musique et vidéo d'art, a été immortalisée sur DVD, étant donné que les huit compositeurs ont créé des musiques sur des films de Nathalie Bujold (Les trains où vont les choses), Chantal DuPont (Pemesu), David Manseau (Agora Obscura) et Leighton Pierce (Number One). Les enregistrements ont été réalisés avec la participation du CIRMMT et les vidéos d'art ont été fournies par Vidéographe 3 . Le DVD comprend aussi le film documentaire Dans les coulisses du Forum 2010, réalisé par Mathieu-David Crépin et Simon Gervais. À travers des extraits de répétitions, des images des activités entourant le Forum (conférences, tables rondes) et des entrevues avec la chef Lorraine Vaillancourt, les musiciens et les compositeurs, ce documentaire de 22 minutes permet d'appréhender l'esprit de ce Forum et d'avoir un aperçu de l'expérience vécue par les participants.

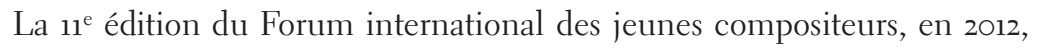
réunissait à Montréal huit compositeurs et permettait d'entrer dans l'univers de la création musicale scandinave. Les participants étaient Zosha Di Castri (Canada), Lauri Mäntysaari (Finlande), Christopher Goddard (Canada), Martin Rane Bauck (Norvège), Frédéric LeBel (Canada), Lisa Streich (Suède), Duncan Schouten (Canada) et Lauri Supponen (Finlande). Leurs œuvres ont toutes été enregistrées et vont paraître sur CD prochainement sous l'étiquette ProNEM. 


\section{Forum 91}

UMMUS / UMM 106 / 1993

Brian Cherney (Canada, 1942)

- Apparitions (1991)

ERIK OÑa (Argentine, 1961)

- Per Ivan Lermoliev

Stefano Gervasoni (Italie, 1962)

- Su un arco di bianco

Mary FinSTERER (Australie, 1962)

- Ruisselant

\section{Forum 93}

UMMUS / UMM 108 / 1995

Melissa Hui (Hong Kong-Canada, 1966)

- Speaking in Tongues

Gediminas Rimkevicius (Lithuanie, 1964)

- Energy, Streams and Directions

Carlos Sanchez-Gutierrez (Mexique, 1964)

- Son del Corazon

\section{Forum 96}

Amberola/UMMUS / AMBC CD $7100 / 1998$

Michael Oesterle (Allemagne-Canada, 1968)

- Vertigo Now

Inouk Demers (Canada, 1970)

- Une pensée de Joseph Knecht au réveil

ElLiott Gyger (Australie, 1968)

- A Wilderness of Mirrors

\section{Forum 98}

Amberola/UMMUS / AMBC CD 7141 / 2000 / double CD

\section{1}

KiRSTY BeILHaRZ (Australie, 1971)

- Burning in the Heart of the Void

Antonio SÉrgio Azevedo (Portugal, 1968)

- Atlas's Journey

Javier Torres Maldonado (Mexique, 1968)

- Exabrupto
CD 2

Yannick Plamondon (Canada, 1970)

- Fil retors

Olga Rayeva (Russie, 1971)

- Die Installation-Landschaft

Joyce W. Brooke (États-Unis, 1973)

- La Quinta del Sordo

\section{Forum 2000-2002}

ATMA Classique / ACD2 2328 / 2003 / double CD

CD1 Forum 2000 - Adelaide

Dominik Karski (Pologne-Australie, 1972)

- Les éruptions du rêve

Brian CurRent (Canada, 1972)

- For the Time Being

Juliana Hodkinson (Angleterre, 1971)

- Some reasons for hesitating

Luis Tinoco (Portugal, 1969)

- Antipode

CD2 Forum 2002 - Montréal

Christopher Tonkin (Australie, 1972)

- Point-Counterpoint

ENeKo Vadillo-Perez (Espagne, 1973)

- Zephyr

Luis Rizo-Salom (Colombie, 1971)

- Al umbral del abismo

LUCA ANTIGNANi (Italie, 1976)

- Il magnifico burattinaio

\section{Forum 2004}

ATMA Classique / ACD2 2375 / 2005/ double CD

Ondrej Adamek (République tchèque, 1979)

- Sinuous Words

Guilherme Carvalho (Brésil, 1974)

- Topologie faible

Du Yun (Chine 1977)

- Impeccable Quake

Derek Johnson (États-Unis, 1976)

- Frozen Light

Julien Bilodeau (Canada, 1974)

- À coups

SAMPO HAAPAMÄKI (Finlande, 1979)

- Fresh

LaURENT Torres (France, 1975)

- Duo Concertante 


\section{Forum 2006 à Amsterdam}

ProNEM / 2008

GEOF HolBrook (Canada, 1978)

- Sets and the Senses

Karola Obermüller (Allemagne, 1977)

- helical

EZEQUiel Menalled (Argentine, 1980)

- $\mathrm{El}$ sistema

Marko Nikodijevic (Serbie-Allemagne, 1980)

- chambres de ténèbres/tombeau de claude vivier

Éric Morin (Canada, 1969)

- Le Coeur serré (2006)

Robin De RaAfF (Pays-Bas, 1968)

- Clarinet Concerto (2006)

\section{Forum 2008 - Musique mixte}

ProNEM / 2009

GRAME - Centre national de création musicale (Lyon)

John Ritz (États-Unis, 1978)

- Nine Secrets

Adam Roberts (États-Unis, 1980)

- Strange Loops

Jimmie LeBlanc (Canada, 1977)

- L'espace intérieur du monde

SÉbastien Béranger (France, 1977)

- Eidétique / Triborder
Forum 2010 - Musique \& vidéo d'art

ProNEM / 2012 / DVD

CIRMMT, Vidéographe

TAYlor Brook (Canada, 1985)

- Les trains où vont les choses

ESAIAS JÄRNEGARD (Suède, 1983)

- Tågen-tiden-tingen

Diego Solfer (Argentine, 1981)

- Trenes

PeiYing Yuan (Singapour, 1984)

- Pemesu

RAFFAele Grimaldi (Italie, 1980)

- Agora Obscura

Hugo Ribeiro (Portugal, 1983)

- Nocturne: rituel

Juan de Dios Magdaleno (Mexique, 1984)

- Yksi (kaksi)

Lu Wang (Chine, 1982)

- Cross-Around 


\section{Collaborations}

- 1992: David Lang «Are You Experienced?»

- 1992: Bali à Montréal

- 1992: Bang on a Can - Live, Volume 1

- 1992 : Barbara Kolb «Millefoglie» and Other Works

- 1995: 9. und 10. Dresdner Tage Der Zeitgenössischen Musik

- 2004: Mary Finsterer - Catch

- 2005 : Javier Torres Maldonado - Exabrupto

- 2005: (Euvres de Jacques Hétu, André Prévost: Yegor Dyachkov

(violoncelle), Jean Saulnier (piano)

- 2007: Portraits de compositeurs canadiens: Alcides Lanza

Le Nouvel Ensemble Moderne apparait comme collaborateur sur neuf CD produits par différents compositeurs, ensembles, interprètes ou festivals. L'ensemble joue alors sur une ou deux pistes de chacun de ces CD.

Des compositeurs montréalais sont à l'honneur sur Bali à Montréal (Claude Vivier et José Evangelista), ainsi que sur le CD du violoncelliste Yegor Dyachkov, qui interprète une œuvre pour violoncelle et ensemble d'André Prévost, inspirée par le grand musicien Yehudi Menuhin. Le NEM apparaît également sur une compilation d'œuvres d'Alcides Lanza présentée dans la collection Portraits de compositeurs canadiens de Centredisques.

Le festival Bang on a Can et le Festival de Dresde ont inclus des enregistrements de prestations du NEM sur leurs scènes dans des productions de CD, soit des œuvres de Julia Wolfe (États-Unis) et Magnus Lindberg (Finlande). Les compositeurs américains David Lang et Barbara Kolb ont aussi collaboré avec le NEM pour immortaliser certaines de leurs œuvres, tout comme Mary Finsterer (Australie) et Javier Torres Maldonado (Mexique), participants respectifs des Forum 1991 et Forum 1998. 


\section{David Lang "Are You Experienced?"}

Composers Recordings Inc. / CD 625/1992

David LaNG (États-Unis, 1957)

- Are You Experienced? (1987)

- Spud (1986)

Jay Rozen (tuba)

David Lang (narrateur)

\section{Bali à Montréal}

UMMUS / UMM 104 / 1992

Claude Vivier (Canada, 1948)

- Pulau Dewata (1977) orchestration de John Rea

José Evangelista (Espagne-Canada, 1943)

- Ô Bali (1989)

Atelier de gamelan de l'Université de Montréal Lorraine et Jean-Eudes Vaillancourt (pianistes)

\section{Bang on a Can - Live, Volume 1}

Composers Recordings Inc. / CD 628 / 1992

Julia Wolfe (États-Unis, 1958)

- The Vermeer Room (1990)

WNWC

Bang on a Can

Barbara Kolb "Millefoglie" and Other Works

New World Record Inc. / NWR 80422-22 / 1992

Barbara Kolb (États-Unis, 1939)

- Millefoglie (1984-1985)

\section{9. und 10. Dresdner Tage Der} Zeitgenössischen Musik

Mittledeutscher Rundfunk / MDR 500-527-12 / 1995

Magnus Lindberg (Finlande, 1958)

- Corrente (1992)

Festival de Dresde

\section{Mary Finsterer - Catch}

ABC Classics / 476 176-o / 2004

Mary Finsterer (Australie, 1962)

- Pascal's Sphere (2000)

\section{Javier Torres Maldonado - Exabrupto}

Stradivarius / STR 33719 / 2005

Javier Torres Maldonado (Mexique, 1968)

- Exabrupto (1998)

QEuvres de Jacques Hétu, André Prévost: Yegor Dyachkov (violoncelle), Jean Saulnier (piano)

Doberman-Yppan / DO 505 / 2005

ANDRÉ Prévost (Canada, 1934-2001)

- Menuhin: Présence (2000)

Yegor DyachKov (violoncelle)

Société Radio-Canada

\section{Portraits de compositeurs canadiens:}

Alcides Lanza

Centredisques / CMCCD 13007 / 2007

Alcides LanZa (Argentine-Canada, 1929)

- aXents (2003)

Centre de musique canadienne 
On ne s'étonne pas que les collaborateurs soient nombreux auprès du NEM. Tous les enregistrements ont été réalisés avec le plus grand soin et l'ensemble joue toujours avec une précision exemplaire. Les interprétations sont toutes de qualité exceptionnelle. Le répertoire endisqué par le NEM a évidemment une grande importance pour la diffusion des œuvres québécoises, mais aussi pour les compositeurs internationaux qui peuvent ainsi mieux se faire connaître ici et ailleurs. Tous les enregistrements effectués dans le cadre des Forums ont largement contribué à l'avancement de la discipline et ont aussi permis de lancer plusieurs carrières. À elles seules, les créations de Forum 91, enregistrées par la Société Radio-Canada, ont donné lieu à plus de cent diffusions radiophoniques à travers le monde.

D'autres projets de disques sont en cours au NEM. Les œuvres créées et enregistrées lors du Forum international des jeunes compositeurs de 2012 seront présentées sur disque comme ceux des éditions précédentes. L’ensemble a déjà terminé l'enregistrement d'un CD monographique comprenant trois œuvres du compositeur d'origine libanaise Zad Moultaka, avec les solistes invités Alexandru Sura (cymbalum) et Pablo Marquez (guitare). On y entendra Où en est la nuit que le NEM a créé au Domaine Forget le 23 août 2013. Ce disque paraîtra sous peu sur l'étiquette L'empreinte digitale dirigée par Catherine Peillon. Pour la saison 2014-2015, l'ensemble prévoit enregistrer des œuvres du compositeur Philippe Leroux.

\section{Les interprètes}

Toujours sous la baguette de Lorraine Vaillancourt, les interprètes du NEM n’ont pas fréquemment été remplacés depuis sa fondation, il y a 25 ans. La qualité des interprétations tient en grande partie à la stabilité de ses effectifs, qui donne une cohésion remarquable à l'ensemble.

Six musiciens fondateurs sont encore actifs au sein du NEM: Michel Bettez (basson), Lise Bouchard (trompette), Jacques Drouin (piano), Normand Forget (hautbois), Alain Giguère (violon) et Julien Grégoire (percussion). La partie de hautbois a été interprétée par Julie Sirois-Leclerc pendant plusieurs années alors que Mark Simons a joué une des parties de clarinette sur les CD Forum 91 et Forum 93. Les autres chaises ont changé une seule fois de titulaire depuis 1989, sauf le trombone (Alain Trudel, Patrice Richer, Angelo Munoz) et le violoncelle (Claude Lamothe, James Darling, Catherine Perron, Julie Trudeau). On note aussi la participation de quelques musiciens invités sur différents enregistrements: Patrick Benoît (tuba), Charles Duquette (percussion), François Gauthier (percussion), Sheila Hannigan (violoncelle), Nadia 
Labelle (cor), Jérôme Laflamme (flûte), Manon Le Comte (harpe), Caroline Lizotte (harpe), Alexander Lozowski (violon), David Martin (trombone), Martin St-Pierre (violon) et Ziya Tabassian (percussion).

\section{Enregistrement du premier CD \\ Benjamin - Gentile - Saariaho - Ligeti}

Salle Claude-Champagne, février 1990

Claude Hamel (violon)

Alain Giguère (violon)

Brian Bacon (alto)

Claude Lamothe (violoncelle)

René Gosselin (contrebasse)

Julien Grégoire (percussion)

Jacques Drouin (piano)

Guy Pelletier (flûte)
Normand Forget (hautbois)

Gilles Plante (clarinette)

André Moisan (clarinette)

Michel Bettez (basson)

Francis Ouellet (cor)

Lise Bouchard (trompette)

Alain Trudel (trombone)

Enregistrement du plus récent CD (* membres fondateurs)

\section{Bouliane - Gougeon - Rea}

Salle Claude-Champagne, janvier 2012

Alain Giguère** (violon)

Johanne Morin (violon)

François Vallières (alto)

Julie Trudeau (violoncelle)

Yannick Chênevert (contrebasse)

Julien Grégoire* (percussion)

Jacques Drouin* (piano)

Jocelyne Roy (flûte)
Normand Forget* (hautbois)

Martin Carpentier (clarinette)

Simon Aldrich (clarinette)

Michel Bettez" (basson)

Jocelyn Veilleux (cor)

Lise Bouchard*" (trompette)

Angelo Munoz (trombone) 


\section{Les chroniques de Circuit}

Plusieurs des disques du NEM ont fait l'objet de chroniques dans les pages de Circuit lors de leur parution. Pour en savoir plus sur ces enregistrements, nous vous invitons donc à reconsulter les numéros correspondants:

Marc Texier: "Chronique des disques québécois: les deux premiers disques du Nouvel Ensemble Moderne», vol. 3, nº 1, 1992, p. 104-107.

. 1991: Benjamin-Gentile - Saariaho - Ligeti

. 1992: Musiques de Montréal

Isabelle Panneton: «Chronique de disques: la musique de Serge Garant», vol. $4, \mathrm{n}^{\circ}$ 1-2, 1993, p. 159-162.

· 1992: Barbara Kolb, «Millefoglie» and Other Works

- 1992: Bali à Montréal

Mark Hyland: "Chronique de disques: Amériques », vol. 4, n 1-2, 1993 , p. $162-163$.

. 1992 : David Lang, «Are You Experienced?»

- 1992: Bang on a Can - Live, Volume I

Michel Gonneville: «Chronique de disques: Forum 91», vol. 5, nº 2, 1994, p. 88-9o.

- 1993: Forum 91

Michel Gonneville: «Chronique de disques: d'ici et d'ailleurs», vol. 6, $\mathrm{n}^{\circ}$ 2, 1995, p. 78-80.

- 1995: Forum 93

Michel Gonneville: «Musiques d'aujourd'hui / Contemporary Music», vol. $9, \mathrm{n}^{\circ}$ 1, 1998, p. 79-82.

- 1996: Jonathan Harvey 2: Bhakti

- 1996: Gougeon - Lesage - Perron - Provost

Cléo Palacio-Quintin : «Nouveautés en bref», vol. 23, nº 1, 2013, p. 86-87.

. 2012: Bouliane-Gougeon-Rea

\section{REMERCIEMENTS}

L'auteure remercie Alain Beauchesne (directeur de production du NEM) qui a fourni de nombreux exemplaires de CD et des informations pour élaborer cette discographie, ainsi que les codirecteurs artistiques Lorraine Vaillancourt et Jacques Drouin qui ont confirmé certains détails. 